\title{
Isolated signed dominating function of graphs
}

\author{
S. Rishitha Dayana ${ }^{1 *}$ and S. Chandra Kumar ${ }^{2}$
}

\begin{abstract}
An isolated signed dominating function(ISDF) of a graph $G$ is a signed dominating function(SDF) $f: V(G) \rightarrow$ $\{-1,+1\}$ such that $f(N[w])=+1$ for at least one vertex of $w \in V(G)$. An isolated signed domination number of $G$, denoted by $\gamma_{i s}(G)$, is the minimum weight of an ISDF of $G$. In this paper, we study some properties of ISDF and we give isolated signed domination number of disconnected graphs, cycles and paths.

Keywords

Isolated domination, signed dominating function, isolated signed dominating function.

\section{AMS Subject Classification}

05 C69.

${ }^{1}$ Research Scholar, Reg No-17223162092029, Department of Mathematics, Scott Christian College, Nagercoil-629003, Tamil Nadu, India. 2 Department of Mathematics, Scott Christian College, Nagercoil-629003, Tamil Nadu, India.

${ }^{*}$ Corresponding author: ${ }^{1}$ rishithadayana@gmail.com

Article History: Received 07 August 2019; Accepted 09 December 2019

(C) 2020 MJM
\end{abstract}

\section{Contents}

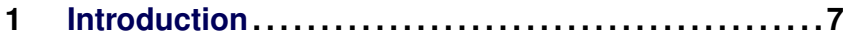

2 Main Results ............................. 8

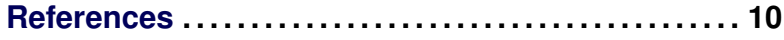

\section{Introduction}

Throughout this paper, we consider only finite, simple and undirected graphs. The set of vertices and edges of a graph $G(p, q)$ will be denoted by $V(G)$ and $E(G)$ respectively, $p=|V(G)|$ and $q=|E(G)|$. For graph theoretic terminology, we follow [7].

For $v \in V(G)$, the open neighborhood of $v$ is $N_{G}(v)=$ $\{u \in V(G): u v \in E(G)\}$ and the closed neighborhood of $v$ is $N_{G}[v]=\{v\} \cup N(v)$. The degree of $v$ is $\operatorname{deg}_{G}(v)=\left|N_{G}(v)\right|$. The minimum and maximum degree of $G$ is defined by $\delta(G)=$ $\min _{v \in V(G)}\{\operatorname{deg}(v)\}$ and $\Delta(G)=\max _{v \in V(G)}\{\operatorname{deg}(v)\}$ respectively. A vertex of degree one is called a pendent vertex. A vertex which is adjacent to a pendent vertex is called a stem.

A function $f: V(G) \rightarrow\{0,1\}$ is called a dominating function if for every vertex $v \in V(G), f(N[v]) \geq 1[8]$.

Various domination functions has been defined from the definition of dominating function by replacing the co-domain $\{0,1\}$ as one of the sets $\{-1,0,1\},\{-1,+1\}$ and etc. One of such example is signed dominating function [3, 4].

In 1995, J.E.Dunbar et al. [4] defined signed dominating function. A function $f: V(G) \rightarrow\{-1,+1\}$ is a signed domi- nating function of $G$, if for every vertex $v \in V(G), f(N[v]) \geq 1$. The signed domination number, denoted by $\gamma_{s}(G)$, is the minimum weight of a signed dominating function on $G$ [4]. The signed dominating function has been studied by several authors including $[1,2,5,6,9,11]$.

A subset $S$ of vertices of a graph $G$ is a dominating set of $G$ if every vertex in $V(G)-S$ has a neighbor in $S$. The minimum cardinality of a dominating set of $G$ is called the domination number and is denoted by $\gamma(G)$.

In 2016, Hameed and Balamurugan [10] introduced the concept of isolate domination in graphs. A dominating set $S$ of a graph $G$ is said to be an isolate dominating set, if $\langle S\rangle$ has at least one isolated vertex [10]. An isolate dominating set $S$ is said to be minimal if no proper subset of $S$ is an isolate dominating set. The minimum and maximum cardinality of a minimal isolate dominating set of $G$ are called the isolate domination number $\gamma_{0}(G)$ and the upper isolate domination number $\Gamma_{0}(G)$ respectively.

By using the definition of signed dominating function and isolate domination, we introduced the concept of isolated signed dominating function. An isolated signed dominating function (ISDF) of a graph $G$ is a function $f: V(G) \rightarrow$ $\{-1,+1\}$ such that $\sum_{u \in N[v]} f(u) \geq 1$ for every vertex $v \in V(G)$ and for at least one vertex of $w \in V(G), f(N[w])=+1$. The weight of $f$, denoted by $w(f)$ is the sum of the values $f(v)$ for all $v \in V(G)$. An isolated signed domination number of $G$, denoted by $\gamma_{i s}(G)$, is the minimum weight of an ISDF of $G$. In this paper, we study some properties of ISDF and we give 
isolated signed domination number of disconnected graphs, cycles and paths.

\section{Main Results}

Lemma 2.1. If a graph $G$ admits ISDF, then $\gamma_{s}(G) \leq \gamma_{i s}(G)$.

Proof. Since every ISDF is a SDF, we have $\gamma_{s}(G) \leq \gamma_{i s}(G)$.

Theorem 2.2. Let $n \geq 2$ be an integer and let $G$ be a disconnected graph with $n$ components $G_{1}, G_{2}, \ldots, G_{n}$ such that the first $r(\geq 1)$ components $G_{1}, G_{2}, \ldots, G_{r}$ admit ISDF. Then $\gamma_{i s}(G)=\min _{1 \leq i \leq r}\left\{t_{i}\right\}$, where $t_{i}=\gamma_{i s}\left(G_{i}\right)+\sum_{j=1, j \neq i}^{n} \gamma_{s}\left(G_{j}\right)$.

Proof. With out loss of generality, we assume that $t_{1}=\min _{1<i<r}\left\{t_{i}\right\}$. Let $f_{1}$ be an minimum ISDF of $G_{1}$ and $f_{i}$ be a minimum SDF of $G_{i}$ for each $i$ with $2 \leq i \leq n$. Then $f: V(G) \rightarrow\{-1,+1\}$ defined by $f(x)=f_{i}(x), x \in V\left(G_{i}\right)$, is an ISDF of $G$ with weight $\gamma_{i s}\left(G_{1}\right)+\sum_{i=2}^{n} \gamma_{s}\left(G_{i}\right)$ and so $\gamma_{i s}(G) \leq$ $\gamma_{i s}\left(G_{1}\right)+\sum_{i=2}^{n} \gamma_{s}\left(G_{i}\right)=t_{1}$.

Let $g$ be a minimum ISDF of $G$. Then there exists an integer $j$ such that $\left.g\right|_{G_{j}}$ is a minimum ISDF of $G_{j}$ for some $j$ with $1 \leq$ $j \leq r$. Also for each $i$ with $1 \leq i \leq n(i \neq j),\left.g\right|_{G_{i}}$ is a minimum SDF of $G_{i}$. Therefore $w(g) \geq \gamma_{i s}\left(G_{j}\right)+\sum_{i=1, i \neq j}^{n} \gamma_{s}\left(G_{i}\right)=t_{j} \geq t_{1}$ and hence $\gamma_{i s}(G)=\min _{1 \leq i \leq r}\left\{t_{i}\right\}$.

Corollary 2.3. Let $H$ be any graph which does not admit ISDF. Then $G=H \cup r K_{1}(r \geq 1)$ admits ISDF with $\gamma_{i s}(G)=$ $r+\gamma_{s}(H)$

Proof. By taking $G_{i} \cong K_{1}$ for $1 \leq i \leq r$ and $G_{r+1} \cong H$ in Theorem 2.2, we can prove the result.

Lemma 2.4. Any odd regular graph does not admit ISDF.

Proof. Since $|N[v]|$ is even, $f(N[v]) \neq 1$ for any $\operatorname{SDF} f: V \rightarrow$ $\{-1,+1\}$.

Lemma 2.5. Let $f$ be an ISDF of $G$ and let $S \subset V$. Then $f(S)=|S|(\bmod 2)$.

Proof. Let $S^{+}=\{v \mid f(v)=1, v \in S\}$ and $S^{-}=\{v \mid f(v)=$ $-1, v \in S\}$. Then $\left|S^{+}\right|+\left|S^{-}\right|=|S|$ and $\left|S^{+}\right|-\left|S^{-}\right|=f(S)$. Therefore $f(S)=|S|-2\left|S^{-}\right|$.

Lemma 2.6. Let $G$ be a graph of order $n$. Then $2 \gamma_{2}(G)-n \leq$ $\gamma_{i s}(G)$.

Proof. Let $f$ be a minimum ISDF of $G$. Let $V^{+}=\{u \in V(G)$ : $f(u)=+1\}$ and $V^{-}=\{v \in V(G): f(v)=-1\}$. If $V^{-}=\phi$, then the proof is clear.

If $v \in V^{-}$since $f(N[v]) \geq 1$, then $v$ has at least two neighbor in $V^{+}$. Therefore $V^{+}$is a 2-dominating set for $G$ and $\left|V^{+}\right| \geq$ $\gamma_{2}(G)$. Since $\gamma_{i s}(G)=\left|V^{+}\right|-\left|V^{-}\right|$and $n=\left|V^{+}\right|+\left|V^{-}\right|$, then $\gamma_{i s}(G)=2\left|V^{+}\right|-n$ and finally we have $\gamma_{i s}(G) \geq 2 \gamma_{2}(G)-$ $n$.
Theorem 2.7. For any graph $G$ with maximum degree $\Delta$ and minimum degree $\delta$, we have $\gamma_{i s}(G) \geq \frac{2+(\delta-\Delta) n}{\Delta+\delta+2}$.

Proof. Let $f$ be a minimum isolated signed domination function of $G$. Then $\left|V^{+}\right|+\left|V^{-}\right|=n$ and $\left|V^{+}\right|-\left|V^{-}\right|=\gamma_{i s}(G)$. We have $\left|V^{+}\right|=\frac{n+\gamma_{i s}(G)}{2}$ and $\left|V^{-}\right|=\frac{n-\gamma_{i s}(G)}{2}$.

By definition of ISDF of $G$, atleast one vertex $v \in V(G)$, we have $f(N[v])=1$. Then $\sum_{v \in V(G)}(d(v)+1) f(v) \geq 1$. Therefore $\sum_{v \in V^{+}}(d(v)) f(v)+\sum_{v \in V^{-}}(d(v)) f(v)+\gamma_{i s}(G) \geq 1$. So $\Delta\left|V^{+}\right|-$ $\delta\left|V^{-}\right|+\gamma_{i s}(G) \geq 1$, thus $\frac{\left(n+\gamma_{i s}(G)\right) \Delta}{2}-\frac{\left(n-\gamma_{i s}(G)\right) \delta}{2}+\gamma_{i s}(G) \geq 1$, we have $\gamma_{i s}(G) \geq \frac{2+(\delta-\Delta) n}{\Delta+\delta+2}$.

Theorem 2.8. For given integer $k \geq 1$, there exists a graph $G$ such that $\gamma_{s}(G)=\gamma_{i s}(G)=k$.

Proof. Let $G=C_{3 k}$ be a cycle of order $3 k$ such that $V(G)=$ $\left\{a_{1}, a_{2}, \ldots, a_{3 k}\right\}$ and $E(G)=\left\{a_{i} a_{i+1}: 1 \leq i \leq 3 k-1\right\}$

$\cup\left\{a_{3 k} a_{1}\right\}$. Let $f$ be a SDF of $G$. Since $N\left[a_{i}\right]=\left\{a_{i-1}, a_{i}, a_{i+1}\right\}$ and $f\left(N\left[a_{i}\right]\right) \geq 1$, any three consecutive vertices must have at least two +1 signs. Thus $f(V(G)) \geq k$. Define a function $f: V(G) \rightarrow\{-1,+1\}$ by

$$
f\left(a_{i}\right)= \begin{cases}-1 & \text { when } i=3 \ell, \ell \geq 1 \\ +1 & \text { otherwise. }\end{cases}
$$

From the above labeling it is easy to observe that $f$ is SDF and $w(f)=k$. Thus $\gamma_{s}(G) \leq k$. The graph $G$ admits ISDF and $\gamma_{i s}(G)=k$ (already proved in Theorem 2.18).

Theorem 2.9. Let $G$ be a connected graph of order $n \geq 2$ in which every vertex is a pendent vertex or stem(we call such graphs as category 1 graph). Then $G$ does not admit ISDF.

Proof. Suppose there exists a SDF of $G$, say ' $f^{\prime}$. Let $u \in$ $V(G)$.

Case 1: If $u$ is pendent vertex, then $f(u)=+1$ (otherwise $f([u]) \leq 0)$.

Case 2: If $u$ is a stem, then $u$ is adjacent with some pendent vertex, say $w$. By Case $1, f(w)=+1$. If $f(u)=-1$, then $f(N[w])=0$. Thus $f(u)=+1$.

Hence $f$ is a constant function with constant +1 . Since $G$ is connected graph of order greater than or equal to $2, f(N[v]) \geq$ 2 for $v \in V(G)$. Thus there exist no vertex $v$ of $G$ such that $f(N[v])=1$, a contradiction.

Corollary 2.10. Let $H$ be any graph and $G=H \circ K_{1}$, then $G$ does not admit ISDF.

Proof. Since every vertex of $G$ is a stem or pendent, the proof follows from Theorem 2.9.

Lemma 2.11. If $G=m K_{1} \cup B$, where $B$ is an union of some graphs from category 1 ( $m \geq 1$ and $B$ may be empty), then $\gamma_{i s}(G)=n$. 
Proof. Let $f$ be an ISDF of $G$ and $u \in V(G)$.

Case 1: If $u \in V\left(m K_{1}\right)$, then $u$ is an isolated vertex ans so $f(u)=+1$.

Case 2: If $u \in V(B)$ then $f(u)=+1$ (as discussed in Theorem 2.9).

Thus $w(f)=n$ and so $\gamma_{i s}(G) \geq n$. But always $\gamma_{i s}(G) \leq n$ and so $\gamma_{i s}(G)=n$.

Lemma 2.12. Suppose $G$ admits ISDF and $\gamma_{i s}(G)=n$. Then $G=m K_{1} \cup B$, where $B$ is an union of some graphs from category 1 ( $m \geq 1$ and $B$ may be empty).

Proof. Let $f$ be an ISDF of $G$. Since $\gamma_{i s}(G)=n, f(v)=+1$ for every $v \in V(G)$.

Suppose there exists no isolated vertex in $G$, then $f(N[v]) \geq 2$ for every $v \in V(G)$, a contradiction to $f$ is an ISDF. Thus $G$ must have an isolated vertex, say $w$. Then $f(N[w])=1$.

Let $H$ be any connected component of $G$ such that $|V(H)| \geq 2$. Suppose $H \notin$ category 1 , then there exists a vertex $v \in V(H)$ such that $v$ is neither pendent nor stem. Then by relabeling the vertices of $V(H)$ by $f(v)=-1$ and $f(w)=1$ for every $w(\neq v) \in V(H)$, we can get a SDF of $H$ such that $f(V(H))<$ $|V(H)|$. Then by Theorem 2.2, $\gamma_{i s}(G)<n$, a contradiction. Thus $H \in$ category 1.

From Lemma 2.11 and Lemma 2.12, we have the following theorem.

Theorem 2.13. Let $G$ be any graph. Then $\gamma_{i s}(G)=n$ if and only if $G \cong m K_{1} \cup B$, where $B$ is an union of some graphs from category 1 ( $m \geq 1$ and $B$ may be empty).

Remark 2.14. Let $G$ be a graph of order $n$ which admits ISDF. Then $\gamma_{i s}(G) \neq n-1$.

Proof. Let $f$ be a minimum ISDF of $G$. Suppose $f(u)=+1$ for all $u \in V(G)$, then $\gamma_{i s}(G)=n$. Suppose $f(u)=-1$ for some $u \in V(G)$, then $\gamma_{i s}(G) \leq n-2$.

Lemma 2.15. Let $G$ be a connected graph of order $n \geq 3$ obtained from $P_{n}$ or $C_{n}$ by adding so many pendent vertices except one vertex of degree two(say $u)$, then $\gamma_{i s}(G)=n-2$.

Proof. Note that except the vertex $u$ all the vertices are either pendent or stem. As discussed in the proof of Theorem 2.9, we must have $f(v)=+1$ for all $v(\neq u) \in V(G)$ and for any ISDF $f$ of $G$. Thus $\gamma_{i s}(G) \geq n-2$.

Define a function $g: V(G) \rightarrow\{-1,+1\}$ by $g(u)=-1$ and $g(v)=+1$ for all $v(\neq u)$. Since $\operatorname{deg}(u)=2, f(N[u])=1$. Also $f(N[v]) \geq 1$ for all $v(\neq u)$. Thus $\gamma_{i s}(G) \leq n-2$.

Example 2.16. The converse part of the above lemma is not true. Consider the following graph $G$. Let $V(G)=\left\{u_{i}\right.$ : $1 \leq i \leq 11\}$ (as given in Figure 1). As discussed in Theorem 2.9, $f\left(u_{i}\right)=+1$ for all $i \neq 2$ and for any ISDF $f$. Suppose $f(u)=+1$, then $f$ is constant function with constant +1 and so $f(N[v]) \geq 2$ for every $v \in V(G)$. Thus $f(u)=-1$ and so $w(f)=n-2$. Thus $\gamma_{i s}(G)=n-2$.

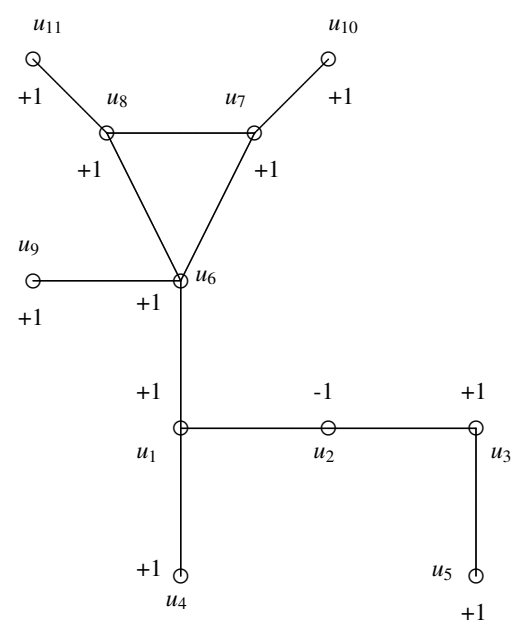

Figure 1: $G$

A Mob $M_{n}(n \geq 1)$ is a tree which is obtained from $P_{4}$, a path on 4 vertices by adding $n$ pendant edges with one end of $P_{4}$.

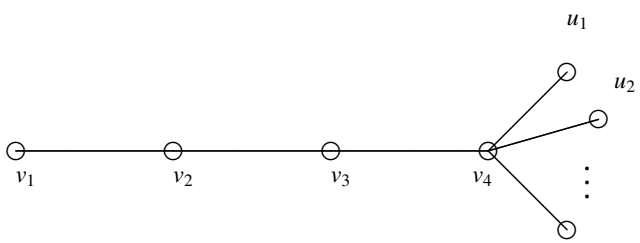

Figure 2: $M_{n}$

$u_{n}$

Lemma 2.17. Let $G=M_{n}$ be a connected graph of order $n \geq 1$ which admits ISDF, then $\gamma_{i s}(G)=n+2$.

Proof. Let $V(G)=\left\{v_{1}, v_{2}, v_{3}, v_{4}, u_{i}: 1 \leq i \leq n\right\}$. Note that except the vertex $v_{3}$ all the vertices $G$ are either pendent or stem. As discussed in the proof of Theorem 2.9, we must have $f(u)=+1$ for all $u\left(\neq v_{3}\right) \in V(G)$ and for any ISDF $f$ of $G$. Thus $\gamma_{i s}(G) \geq n-2$.

Define a function $g: V(G) \rightarrow\{-1,+1\}$ by $g\left(v_{3}\right)=-1$ and $g(u)=+1$ for all $u\left(\neq v_{3}\right)$. Since $\operatorname{deg}\left(v_{3}\right)=2, f\left(N\left[v_{3}\right]\right)=1$. Also $f(N[u]) \geq 1$ for all $u\left(\neq v_{3}\right)$. Thus $\gamma_{i s}(G) \leq n+2$.

Lemma 2.18. Let $n \geq 3$ be an integer. Then the cycle $C_{n}$ admits ISDF with ISDN

(1) $\gamma_{i s}\left(C_{n}\right)=k$ when $n=3 k$.

(2) $\gamma_{i s}\left(C_{n}\right)=k+1$ when $n=3 k+1$.

(3) $\gamma_{i s}\left(C_{n}\right)=k+2$ when $n=3 k+2$.

Proof. Let $n \geq 3$ be an integer. Let $V\left(C_{n}\right)=\left\{a_{i} / 1 \leq i \leq n\right\}$ and $E\left(C_{n}\right)=\left\{a_{i} a_{i+1} / 1 \leq i \leq n-1\right\} \cup\left\{a_{n} a_{1}\right\}$. Let $f$ be an ISDF. Since $N\left[a_{i}\right]=\left\{a_{i-1}, a_{i}, a_{i+1}\right\}$ and $f\left(N\left[a_{i}\right]\right) \geq 1$, any three consecutive vertices must have at least two +1 signs. (1)

Case 1: Suppose $n=3 k$. Then by (1), $f(V(G)) \geq k$.

Case 2: Suppose $n=3 k+1$. Suppose $f\left(a_{3 k+1}\right)=-1$. Then by (1), we get $f\left(\left\{a_{3 k+1}, a_{1}, a_{2}\right\}\right) \geq 1, f\left(\left\{a_{3}, a_{4}, a_{5}\right\}\right) \geq 1$, 
$f\left(\left\{a_{6}, a_{7}, a_{8}\right\}\right) \geq 1, \ldots, f\left(\left\{a_{3(k-1)}, a_{3 k-2}, a_{3 k-1}\right\}\right) \geq 1$. Suppose $f\left(a_{3 k}\right)=-1$ then $f\left(\left\{a_{3 k}, a_{3 k+1}, a_{1}\right\}\right) \leq-1$, a contradiction to (1). Thus $f\left(a_{3 k}\right)=-1$ and so $f(V(G)) \geq k+1$.

Similarly, we can get $f(V(G)) \geq k+1$ when $f\left(a_{3 k+1}\right)=+1$. Case 3: Suppose $n=3 k+2$. By (1) both $f\left(a_{3 k+1}\right)$ and $f\left(a_{3 k+2}\right)$ are not simultaneously equal to -1 .

Suppose $f\left(a_{3 k+1}\right)=+1$ and $f\left(a_{3 k+2}\right)=+1$, then by (1) we can get $f(V(G)) \geq k+2$.

Suppose $f\left(a_{3 k+2}\right)=-1$. Then by (1), we get $f\left(\left\{a_{3 k+2}, a_{1}, a_{2}\right\}\right.$ )$\geq 1, f\left(\left\{a_{3}, a_{4}, a_{5}\right\}\right) \geq 1, f\left(\left\{a_{6}, a_{7}, a_{8}\right\}\right) \geq 1, \ldots, f\left(\left\{a_{3(k-1)}\right.\right.$, $\left.\left.a_{3 k-2}, a_{3 k-1}\right\}\right) \geq 1$. Suppose $f\left(a_{3 k}\right)=-1$ or $f\left(a_{3 k+1}\right)=$ -1 then $f\left(\left\{a_{(3 k)}, a_{3 k+1}, a_{3 k+2}\right\}\right) \leq-1$, a contradiction to (1). Thus $f\left(a_{3 k}\right)=-1$ and so $f(V(G)) \geq k+2$. Similarly, we can get $f(V(G)) \geq k+2$ when $f\left(a_{3 k+2}\right)=+1$. Define a function $f: V\left(C_{n}\right) \rightarrow\{-1,+1\}$ by

$$
f\left(a_{i}\right)= \begin{cases}-1 & \text { when } i=3 \ell, \ell \geq 1 \\ +1 & \text { otherwise } .\end{cases}
$$

Define From the above labeling it is easy to observe that $f$ is ISDF. Also $\gamma_{i s}\left(C_{3 k}\right) \leq k, \gamma_{i s}\left(C_{3 k+1}\right) \leq k+1$ and $\gamma_{i s}\left(C_{3 k+2}\right) \leq$ $k+2$.

Lemma 2.19. Let $n \geq 6$ and $k \geq 2$ be an integers. Then the path $P_{n}$ admits ISDF with ISDN

(1) $\gamma_{i s}\left(P_{n}\right)=k+2$ when $n=3 k$.

(2) $\gamma_{i s}\left(P_{n}\right)=4+(k-1)$ when $n=3 k+1$.

(3) $\gamma_{i s}\left(P_{n}\right)=2+k$ when $n=3 k+2$.

Proof. Let $n \geq 6$ be an integers. Let $V\left(P_{n}\right)=\left\{a_{i} / 1 \leq i \leq n\right\}$ and $E\left(P_{n}\right)=\left\{a_{i} a_{i+1} / 1 \leq i \leq n-1\right\}$.

Claim 1: If $f\left(a_{1}\right)=+1$, then $f\left(a_{2}\right)=+1$.

Suppose $f\left(a_{2}\right)=-1$, then $f\left(N\left[a_{1}\right]\right) \leq 0$, a contradiction to $f$ is ISDF.

Similarly, we can prove that $f\left(a_{n-1}\right)=+1$, then $f\left(a_{n}\right)=+1$. Let $f$ be an ISDF. Since $N\left[a_{i}\right]=\left\{a_{i-1}, a_{i}, a_{i+1}\right\}$ for $2 \leq i \leq$ $n-1$ and $f\left(N\left[a_{i}\right]\right) \geq 1$, any three consecutive vertices must have at least two +1 signs. - (1)

Case 1: Suppose $n=3 k$. Then by Claim 1, we get $f\left(a_{1}\right)=$ $f\left(a_{2}\right)=f\left(a_{3 k-1}\right)=f\left(a_{3 k}\right)=+1$. Suppose $f\left(a_{3 i}\right)=-1$ for some $i=1,2, \ldots, k$. Choose $j$ be the largest integer there exists $i, f\left(a_{3 j}\right)=-1$ for $1 \leq j \leq k-1$. Since $f\left(N\left[a_{3 j}\right]\right) \geq 1$, we have $f\left(a_{3 j+1}\right)=f\left(a_{3 j+2}\right)=+1$. By the definition of $j$, $f\left(a_{3 j+3}\right)=+1$. Now $f\left(\left\{a_{1}, a_{2}, a_{3}\right\}\right) \geq 1, f\left(\left\{a_{4}, a_{5}, a_{6}\right\}\right) \geq 1$, $\ldots, f\left(\left\{a_{3 j-2}, a_{3 j-1}, a_{3 j}\right\}\right) \geq 1, f\left(\left\{a_{3 j+1}, a_{3 j+2}, a_{3(j+1)}\right\}\right) \geq$ $3, f\left(\left\{a_{3(j+1)+1}, a_{3(j+1)+2}, a_{3(j+2)}\right\}\right) \geq 1, \ldots, f\left(\left\{a_{3 k-2}, a_{3 k-1}\right.\right.$, $\left.\left.a_{3 k}\right\}\right) \geq 1$. Thus $f(V(G)) \geq j+3+(k-j-1)$ and hence $f(V(G)) \geq k+2$.

Case 2: Suppose $n=3 k+1$. Then by Claim 1, we get $f\left(a_{1}\right)=f\left(a_{2}\right)=f\left(a_{3 k}\right)=f\left(a_{3 k+1}\right)=+1$. Suppose $f\left(a_{3 i}\right)=$ -1 for some $i=1,2, \ldots, k$. Choose $j$ be the largest integer there exists $i, f\left(a_{3 j}\right)=-1$ for $1 \leq j \leq k-1$. Since $f\left(N\left[a_{3 j}\right]\right) \geq 1$, we have $f\left(a_{3 j+1}\right)=f\left(a_{3 j+2}\right)=+1$. By the definition of $j, f\left(a_{3 j+3}\right)=+1$. Now $f\left(\left\{a_{1}, a_{2}, a_{3}\right\}\right) \geq 1$, $f\left(\left\{a_{4}, a_{5}, a_{6}\right\}\right) \geq 1, \ldots, f\left(\left\{a_{3 j-2}, a_{3 j-1}, a_{3 j}\right\}\right) \geq 1$, $f\left(\left\{a_{3 j+1}, a_{3 j+2}, a_{3(j+1)}\right\}\right) \geq 3, f\left(\left\{a_{3(j+1)+1}, a_{3(j+1)+2}\right.\right.$, $\left.\left.a_{3(j+2)}\right\}\right) \geq 1, \ldots, f\left(\left\{a_{3 k-1}, a_{3 k}, a_{3 k+1}\right\}\right) \geq 1$. Thus $f(V(G)) \geq$ $j+3+(k+1-j-1)$ and hence $f(V(G)) \geq k+3$.

Define a function $f: V\left(P_{n}\right) \rightarrow\{-1,+1\}$ by

$$
f\left(a_{i}\right)= \begin{cases}-1 & \text { when } i=3 \ell, 1 \leq \ell \leq k-1 \\ +1 & \text { otherwise }\end{cases}
$$

From the above labeling it is easy to observe that $f$ is ISDF. Also $\gamma_{i s}\left(P_{3 k}\right) \leq k+2$ and $\gamma_{i s}\left(P_{3 k+1}\right) \geq k+3$.

Case 3: suppose $n=3 k+2$ for $k \geq 2$. Then by Claim 1, we get $f\left(a_{1}\right)=f\left(a_{2}\right)=f\left(a_{3 k+1}\right)=f\left(a_{3 k+2}\right)=+1$ and by (1), we get $f(V(G)) \geq k+2$. Define a function $f: V\left(P_{n}\right) \rightarrow$ $\{-1,+1\}$ by

$$
f\left(a_{i}\right)= \begin{cases}-1 & i=3 \ell, 1 \leq \ell \leq k \\ +1 & \text { otherwise }\end{cases}
$$

From the above labeling it is easy to observe that $f$ is ISDF. Also $\gamma_{i s}\left(P_{3 k+2}\right) \leq k+2$.

\section{References}

[1] G. J. Chang, S. C. Liaw, H. G. Yeh, k-Subdomination in graphs, Discrete Appl. Math. 120 (2002), 44-60.

[2] E. J. Cockayne and C. M. Mynhardt, On a generalization of signed dominating functions of graphs, Ars Combin., 43 (1996), 235-245.

[3] J. E. Dunbar, S. T. Hedetniemi, M. A. Henning, and A. A. McRae. Minus domination in regular graphs. Discrete Math., 149 (1996), 311-312.

[4] J. E. Dunbar, S. T. Hedetniemi, M. A. Henning and P. J. Slater, Signed domination in graphs. In: Graph Theory, Combinatorics and Applications. Proc. 7th Internat. conf. Combinatorics, Graph Theory, Applications, (Y. Alavi, A. J. Schwenk, eds.). John Wiley \& Sons, Inc., 1 (1995), 311-322.

[5] O. Favaron, Signed domination in regular graphs, Discrete Math., 158 (1996), 287-293.

[6] Z. Füredi and D. Mubayi, Signed domination in regular graphs and set-systems, J. Combin. Theory Series B,76 (1999), 223-239.

[7] F. Harary, Graph Theory, Addison-Wesley, (1969).

[8] T. W. Haynes, S. T. Hedetniemi and P. J. Slater "Fundamental of domination in graphs". Marcel Dekker inc...... New York-Basel-Hong Kong, (1998).

[9] Huaming Xing, Langfang, Liang Sun, Beijing, and Xuegang Chen, Taian, On signed distance-k-domination in graphs, Czechoslovak Mathematical Journal,56(131) (2006), 229-238.

[10] I. Sahul Hameed, S. Balamurugan, Isolate domination in graphs, Arab J Math Sci., 22 (2016), 232-241. 
[11] Z. Zhang, B. Xu, Y. Li and L. Liu, A note on the lower bounds of signed domination number of a graph, Discrete Math., 195 (1999), 295-298.

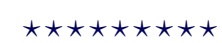

$\operatorname{ISSN}(\mathrm{P}): 2319-3786$

Malaya Journal of Matematik

$\operatorname{ISSN}(\mathrm{O}): 2321-5666$

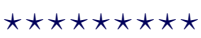

